\title{
Thirty-Day Post-Discharge Outcomes Following COVID-19 Infection
}

Justin R. Kingery, MD/PhD ${ }^{1,2}$ (D), Paul BF Martin, $M D^{1}$, Ben R. Baer, $M S^{3}$, Laura C. Pinheiro, $\mathrm{PhD}^{7}$, Mangala Rajan, $\mathrm{MBA}^{7}$, Adrienne Clermont, $\mathrm{MSPH}^{4}$, Sabrina Pan, BS4, Khoi Nguyen, BA ${ }^{4}$, Khalid Fahoum, MSc ${ }^{4}$, Graham T. Wehmeyer, MD ${ }^{4}$, Mark N. Alshak, MD4, Han A. Li, MD', Justin J. Choi, MD ${ }^{7}$, Martin F. Shapiro, MD', Margaret L. McNairy, MD/MSc ${ }^{1,2}$, Monika M. Safford, MD' , and Parag Goyal, MD ${ }^{1,5}$

'Division of General Internal Medicine, Department of Medicine, Weill Cornell Medicine, New York, NY, USA; ${ }^{2}$ Center for Global Health, Department of Medicine, Weill Cornell Medicine, New York, NY, USA; ${ }^{3}$ Department of Statistics and Data Science, Cornell University, Ithaca, NY, USA; ${ }^{4}$ School of Medicine, Weill Cornell Medicine, New York, NY, USA; ${ }^{5}$ Division of Cardiology, Department of Medicine, Weill Cornell Medicine, New York, NY, USA.

BACKGROUND: The clinical course of COVID-19 includes multiple disease phases. Data describing post-hospital discharge outcomes may provide insight into disease course. Studies describing post-hospitalization outcomes of adults following COVID-19 infection are limited to electronic medical record review, which may underestimate the incidence of outcomes.

OBJECTIVE: To determine 30-day post-hospitalization outcomes following COVID-19 infection.

DESIGN: Retrospective cohort study

SETTING: Quaternary referral hospital and community hospital in New York City.

PARTICIPANTS: COVID-19 infected patients discharged alive from the emergency department (ED) or hospital between March 3 and May 15, 2020.

MEASUREMENT: Outcomes included return to an ED, re-hospitalization, and mortality within 30 days of hospital discharge.

RESULTS: Thirty-day follow-up data were successfully collected on $94.6 \%$ of eligible patients. Among 1344 patients, $16.5 \%$ returned to an $\mathrm{ED}, 9.8 \%$ were re-hospitalized, and $2.4 \%$ died. Among patients who returned to the ED, 50.0\% (108/216) went to a different hospital from the hospital of the index presentation, and 61.1\% (132/ 216) of those who returned were re-hospitalized. In Cox models adjusted for variables selected using the lasso method, age (HR 1.01 per year [95\% CI 1.00-1.02]), diabetes (1.54 [1.06-2.23]), and the need for inpatient dialysis (3.78 [2.23-6.43]) during the index presentation were independently associated with a higher re-hospitalization rate. Older age (HR 1.08 [1.05-1.11]) and Asian race (2.89 [1.27-6.61]) were significantly associated with mortality. CONCLUSIONS: Among patients discharged alive following their index presentation for COVID-19, risk for returning to a hospital within 30 days of discharge was substantial. These patients merit close post-discharge follow-up to optimize outcomes.

KEY WORDS: COVID-19; mortality; re-admission; discharge.

Justin R. Kingery and Paul Martin contributed equally to this work. Received January 4, 2021

Accepted May 6, 2021

Published online June 7, 2021
$\mathrm{J}$ Gen Intern Med 36(8):2378-85

DOI: $10.1007 / \mathrm{s} 11606-021-06924-0$

(c) Society of General Internal Medicine 2021

\section{INTRODUCTION}

The burden of the novel coronavirus disease (COVID-19) is increasing with more than 70 million cases and 1.6 million deaths as of November $2020,{ }^{1}$ with a cost of nearly $\$ 17$ trillion in the USA alone in $2020 .^{2}$ While many studies have examined inhospital outcomes, ${ }^{3-5}$ the consequences of COVID-19 likely extend beyond the hospitalization period. ${ }^{6-8}$ Similar to other conditions, ${ }^{19-11}$ it is likely that the first 30 days following hospital discharge are an especially vulnerable period for adverse outcomes. Yet, few studies to date have focused on posthospitalization outcomes in COVID-19; and among these studies, only post-hospitalization outcomes obtained by electronic medical record system abstraction have been captured, raising concern for underestimation of adverse outcomes. ${ }^{12-20}$ Therefore, the actual incidence of COVID-associated post-discharge return to the emergency room, re-hospitalization, and mortality remains unknown.

To address this important gap in knowledge, we conducted a retrospective study of 30-day post-discharge outcomes among adults discharged alive from our hospital system in New York City following a symptomatic confirmed COVID19 infection. The objectives of this study were to determine the incidence of return to an emergency room, re-hospitalization, and mortality in the first 30 days after discharge from the ED or hospital, and to identify determinants of these outcomes.

\section{METHODS}

\section{Study Design}

This was a retrospective observational cohort study of adults from a COVID-19 clinical registry. ${ }^{21}$ 


\section{Study Population}

We examined consecutive adults aged at least 18 years who presented with acute COVID-19 to either New York-Presbyterian/Weill Cornell Medical Center (NYP/WCMC) or Lower Manhattan Hospital (NYP/LMH) in New York City between March 3 (date of the first case) and May 15, 2020 (which was the peak of the first wave of the COVID-19 pandemic in the region); and were subsequently discharged alive from either the ED or following a hospitalization. As has been previously described, ${ }^{21}$ cases were confirmed to have COVID-19 through reverse-transcriptase-polymerase-chain-reaction (RT-PCR) assays performed on nasopharyngeal specimens. We included the index presentation for each patient. Patients discharged to hospice services were excluded.

\section{Study Setting}

$\mathrm{NYP} / \mathrm{WCMC}$ is an 862-bed quaternary referral center located in the Upper East Side of Manhattan, New York; and NYP/ LMH is an affiliated 180-bed community hospital located in the Lower East Side of Manhattan, NY.

\section{Data Collection}

Study procedures for this cohort have been previously described. ${ }^{21}$ Briefly, chart abstraction was conducted by trained personnel who recorded parameters related to demographics, medical history, symptoms, imaging and laboratory values, inhospital complications, in-hospital treatment, and length of stay. Data were entered into a RedCap database. ${ }^{22}$ Prior evaluation of inter-abstractor agreement was high $(\mathrm{r}=0.92)$.

\section{Outcomes}

The main study outcomes were as follows: 1) return to any ED, 2) re-hospitalization, and 3) death within 30 days of discharge. To collect these data, we contacted patients by phone at least 30 days after the discharge date. Specifically, we called patients up to six times at different times of the day, over a 2-week period. For patients who did not answer our attempted calls, we contacted the listed healthcare proxy to ascertain whether the patient had experienced any outcome of interest. If an event occurred, we inquired about the event date. Patients were considered lost-to-follow-up if we were unable to ascertain data from either the patient or their healthcare proxy after these attempts.

\section{Statistical Analysis}

We calculated medians with interquartile ranges for continuous variables and proportions for categorical variables. To identify independent determinants of each outcome, we created separate models for each of the three outcomes using the following multi-step procedure. First, we used the statistical method Lasso to generate a sequence of models which best fit the data. ${ }^{23}$ Lasso finds the best models for the data with a given sum of absolute values of the regression coefficients. By forcing the overall size of the coefficients to be small through regularization, this method zeroes out coefficients for less important variables, and thus selects a subset of variables considered important for the model. We then chose the model which best satisfied the assumptions among the models nearby the model given by the one-standard-error rule for tuning parameter selection. Variables from these models were then inputted into a multivariable Cox proportional hazard model (without regularization).

Variables under consideration for selection included socio-demographics (age, sex, race, housing status), length of stay, comorbidities (tobacco use, body mass index $[\mathrm{BMI}]$, cardiovascular disease, chronic kidney disease, diabetes, chronic lung disease, HIV, active cancer, and other immunocompromised state), symptoms (fever, cough, diarrhea, nausea/vomiting, myalgia, dyspnea), and chest imaging at presentation; clinical course (new-onset myocardial infarction/arrhythmia/heart failure, dialysis, vasopressor requirement, hypoxia upon presentation, intubation/extubation, and tracheostomy) and infection parameters (PCR viral load, respiratory viral pathogen panel, and blood culture). Of note, separate analyses including either length of stay, days from symptom onset to admission, or days from symptom onset to discharge were performed and yielded similar results. Therefore, we included the conventional variable, length of stay, in this analysis. PCR viral load was based on cycle threshold $(\mathrm{Ct})$ value, which represents the number of replication cycles required for sufficient gene amplification to produce a fluorescent signal that crosses a predefined threshold. Based on prior work that defined viral load according to terciles and demonstrated a dose-response relationship with mortality, low, medium, and high viral loads were defined as PCR cycle threshold $>30,25-30$, and $<25$ cycles, respectively. ${ }^{24}$

We used multivariate imputation by chained equations (MICE) to account for missing data. ${ }^{25,}{ }^{26}$ Missingness was highest for race (13\% missing) and BMI (7\% missing); the remaining variables had $<1 \%$ missing. All models were tested to ensure none of the variables violated the proportional hazard assumptions using Schoenfeld residuals. The low mortality rate in this cohort precluded the need to consider death as a competing risk. To determine statistical significance, we used 2 -sided hypothesis testing with a $p$-value $<0.05$. These analyses were performed using R version 3.5.1 software (R Foundation, Vienna, Austria).

\section{ETHICAL CONSIDERATIONS}

This study was approved by the institutional review board of Weill Cornell Medicine (IRB: 20-03021681) and granted a waiver of informed consent. 


\section{RESULTS}

\section{Study Population}

Between March 3 and May 15, 2020, 1935 patients presented to either WCMC or LMH (Fig. 1). As of June 15, 2020, 287 $(14.8 \%)$ died during the index hospitalization, $183(9.5 \%)$ remained hospitalized, $8(0.4 \%)$ were discharged to hospice care, and 36 (1.9\%) were transferred to outside hospitals for which we did not have access to medical records. Among the 1421 patients eligible for this study, we collected 30-day follow-up data on 1344 patients (94.6\% of eligible patients).

\section{Characteristics of the Study Population}

The baseline characteristics of this cohort are shown in Table 1. The median age was 61.0 years (IQR 48-72), and $44.5 \%$ were female. Common comorbid conditions were hypertension (51.2\%), diabetes $(28.3 \%)$, and chronic kidney disease $(9.1 \%)$. The majority of patients presented with fever (71.4\%), cough $(72.1 \%)$, or dyspnea $(61.2 \%)$. Of those who received a respiratory viral panel PCR swab, 1.9\% (6/312) were positive for a viral co-infection. Initial chest radiograph revealed bilateral pulmonary infiltrates in $60.1 \%$ of patients.

Characteristics stratified by whether the patient was discharged alive from the ED or following hospitalization are shown in Supplemental Table 1. Among patients discharged alive from the $\operatorname{ED}(15.0 \%, 201 / 1344)$, the median age was 49 years (IQR 48-72), 48.3\% were male, and the median BMI was $28 \mathrm{~kg} / \mathrm{m}^{2}$ (IQR 24-32). Most presented from home (90.5\%), and $24.9 \%$ were healthcare workers. The most common comorbid conditions were hypertension $(34.8 \%)$, diabetes $(16.4 \%)$, and coronary artery disease $(7.5 \%)$. Six patients $(3.0 \%)$ required supplemental oxygen by nasal cannula. Fever (73.1\%), cough $(71.1 \%)$, and dyspnea $(51.2 \%)$ were the most common presenting symptoms. Bilateral infiltrates on chest radiograph were present in $20.4 \%$.

Among the patients discharged alive following hospitalization $(85.0 \%, 1143 / 1344)$, median age was 63.0 years (IQR 5073 ), $43 \%$ were female, and the median BMI was $27 \mathrm{~kg} / \mathrm{m}^{2}$ (IQR 24-32). The most common comorbid conditions were hypertension $(54 \%)$, diabetes $(30.4 \%)$, and coronary artery disease (12.6\%). On admission, fever (71.1\%), cough $(72.3 \%)$, and dyspnea $(62.9 \%)$ were the most common presenting symptoms. Nearly half $(45.4 \%)$ required supplemental oxygen within the first $3 \mathrm{~h}$ of presentation-34.5\% required nasal cannula, $9.1 \%$ required noninvasive ventilation, and $1.8 \%$ required mechanical ventilation. During the hospital stay, $12.1 \%$ were intubated at a median of 1.5 days (IQR 1.00-3.75) after admission, and $12 \%$ required vasopressors. Arrhythmia (4.4\%), heart failure $(2.2 \%)$, and myocardial infarction $(2.1 \%)$ occurred infrequently.

\section{Outcomes}

Among the full cohort of 1344 patients, 16.5\% (216/1,344) returned to an ED, $9.8 \%$ (132/1344) were re-hospitalized, and $2.4 \%$ (32/1344) died within 30 days of discharge. KaplanMeier analysis is shown in Figure 2. The median time from discharge to return to an ED was 6.2 days (IQR 2.3-16.8), median time to re-hospitalization was 5.4 days (IQR 1.913.5), and median time to death was 9.3 days (IQR 5.414.5). Fifty percent of patients (108/216) who returned to the emergency room went to a different hospital from that of the index presentation.

Among patients who returned to the ED, 61.1\% (132/216) were re-hospitalized. Among patients who returned to the ED but were not hospitalized, the mortality rate was $1.5 \%$; and among patients who were hospitalized, the mortality rate was

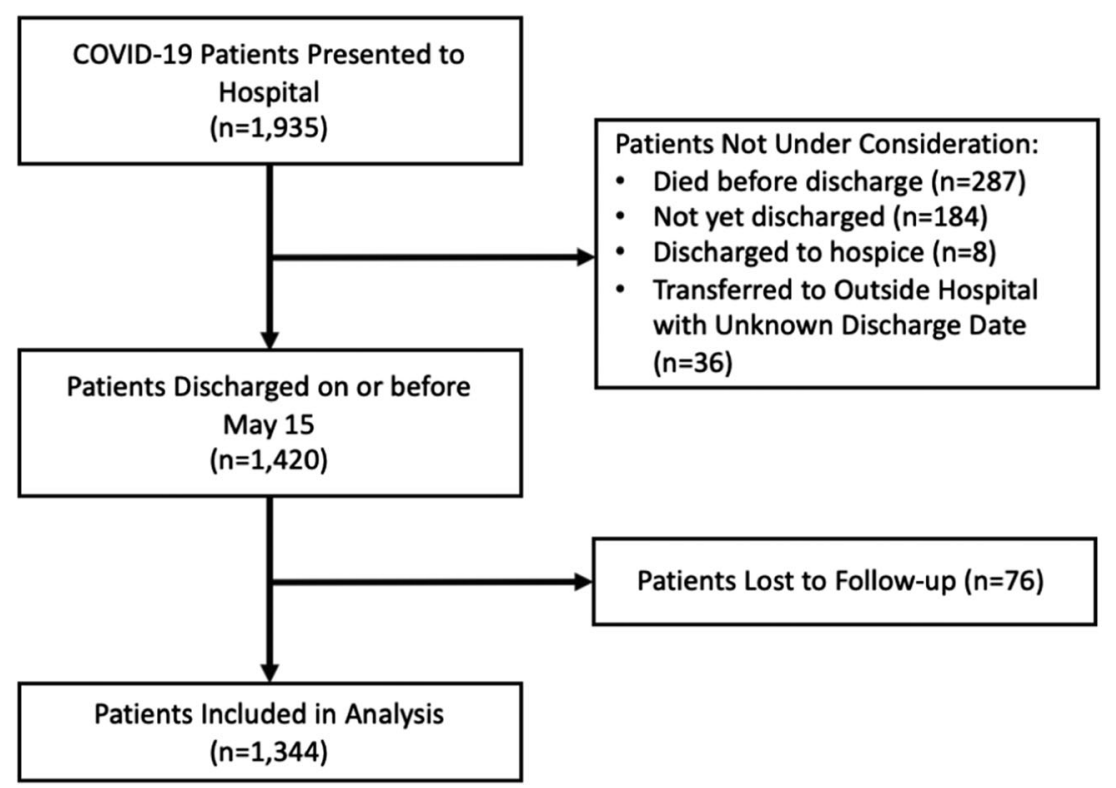

Figure 1 Exclusion cascade describing COVID-19-infected patients included in the analysis. 
Table 1 Baseline Characteristics of Cohort

\begin{tabular}{|c|c|}
\hline $\begin{array}{l}\text { Study participants } \\
(n=1344)\end{array}$ & $\begin{array}{l}\text { Variable } \\
\text { n }(\%) \\
\text { Mean (SD) }\end{array}$ \\
\hline \multicolumn{2}{|l|}{ Patient demographics } \\
\hline Age (years) & $61.0(48-72)$ \\
\hline Female & $598(44.5 \%)$ \\
\hline Race & $27.6(5.9)$ \\
\hline Asian & $211(15.7 \%)$ \\
\hline Black & $202(15.0 \%)$ \\
\hline White & $454(33.8 \%)$ \\
\hline Other & $301(22.4 \%)$ \\
\hline \multirow{2}{*}{ Body mass index $\left(\mathrm{kg} / \mathrm{m}^{2}\right)$} & $176(13.1 \%)$ \\
\hline & $27(24-32)$ \\
\hline \multirow{2}{*}{\multicolumn{2}{|c|}{ Healthcare worker }} \\
\hline & Admitted from: \\
\hline Home & $1206(89.7 \%)$ \\
\hline Nursing/Rehab Facility & $65(4.8 \%)$ \\
\hline Undomiciled & $24(1.8 \%)$ \\
\hline \multicolumn{2}{|l|}{ Health behaviors/comorbidities } \\
\hline \multicolumn{2}{|l|}{ Past medical history } \\
\hline Diabetes & $381(28.3 \%)$ \\
\hline Hypertension & $688(51.2 \%)$ \\
\hline COPD & $52(3.9 \%)$ \\
\hline Chronic kidney disease & $47(3.5 \%)$ \\
\hline ESRD & $75(5.6 \%)$ \\
\hline Cirrhosis & $16(1.2 \%)$ \\
\hline Coronary artery disease & $159(11.8 \%)$ \\
\hline Heart failure & $72(5.4 \%)$ \\
\hline Cancer & $71(5.3 \%)$ \\
\hline HIV & $27(2.0 \%)$ \\
\hline Immunosuppression & $24(1.8 \%)$ \\
\hline Current tobacco/vape use & $236(17.6 \%)$ \\
\hline Presenting symptoms/hospital course & \\
\hline Dyspnea & $822(61.2 \%)$ \\
\hline Fever & $960(71.4 \%)$ \\
\hline Cough & $969(72.1 \%)$ \\
\hline Diarrhea & $361(26.9 \%)$ \\
\hline Nausea or vomiting & $274(20.4 \%)$ \\
\hline Myalgias & $315(23.4 \%)$ \\
\hline Supplemental $\mathrm{O}_{2}$ within first $3 \mathrm{~h}$ & \\
\hline No oxygen required & $817(60.8 \%)$ \\
\hline Nasal cannula & $400(29.9 \%)$ \\
\hline Noninvasive/BiPAP & $106(7.9 \%)$ \\
\hline Mechanical ventilation & $21(1.6 \%)$ \\
\hline Initial chest radiograph & \\
\hline Normal & $364(27.1 \%)$ \\
\hline Unilateral infiltrates & $172(12.8 \%)$ \\
\hline Bilateral infiltrates & $808(60.1 \%)$ \\
\hline Positive respiratory viral panel* & $6(0.4 \%)$ \\
\hline Positive blood culture & $42(3.1 \%)$ \\
\hline During hospitalization: & \\
\hline Myocardial infarction & $24(1.8 \%)$ \\
\hline New arrhythmia & $50(3.7 \%)$ \\
\hline New heart failure & $25(1.9 \%)$ \\
\hline Dialysis requirement & $61(4.5 \%)$ \\
\hline Required intubation & $138(10.3 \%)$ \\
\hline Extubated or tracheostomy & $20(1.5 \%)$ \\
\hline Discharged from ED & $201(15.0 \%)$ \\
\hline Days from symptom onset to admission & $7(3-10)$ \\
\hline Days from symptom onset to discharge & $14(7-21)$ \\
\hline Length of hospital stay (if admitted) & $6(3-12)$ \\
\hline
\end{tabular}

COPD, chronic obstructive pulmonary disease; ESRD, end-stage renal disease; BiPAP, bilevel positive airway pressure

*Positive for viral pathogen in addition to COVID-19

$2.5 \%$. Among patients who died, nearly half $(46.9 \%)$ died without re-presenting to the hospital.

Of note, patients discharged after hospitalization were more likely to return to the $\mathrm{ED}(23.4$ vs. $14.8 \%, p=0.002)$ and numerically more likely to be re-hospitalized $(12.4 \%$ vs. $9.4 \%, p=0.18)$ compared to those discharged from the ED. Mortality rates were similar in these groups $(1.5 \%$ vs. $2.5 \%, p=0.37)$.

\section{Factors Associated with Return to the Emergency Room}

The Lasso model selected the following variables as important for return to the ED: age, sex, undomiciled status, need for dialysis, bilateral infiltrates on presenting chest radiograph, and discharge following hospitalization. Based on a univariate Cox proportional hazard model, age, sex, undomiciled status, and need for dialysis were associated with return to the emergency room; and bilateral infiltrates and discharge from inpatient hospitalization were inversely associated with return to the emergency room. (Supplemental Table 2).

In a multivariable Cox proportional hazard regression analysis, older age (HR 1.007 per year [95\% CI 1.00-1.02]), undomiciled status (5.13 [2.83-9.30]), and need for dialysis during index hospitalization (3.59 [2.29-5.65]) were significantly associated with returning to the ED within 30 days of discharge; and bilateral chest radiograph infiltrate on presenting chest radiograph $(0.46$ [0.33-0.63]) was inversely associated with returning to the ED within 30 days of discharge (Table 2). In a sensitivity analysis including a subset of 970 patients with available COVID-19 PCR cycle threshold, the multivariable Cox proportional hazard regression analysis revealed similar results (Supplemental Table 3). Additionally, high cycle threshold (i.e., low viral load) was associated with a trend toward higher risk of return to the emergency room (1.36 [0.95-1.95]).

\section{Factors Associated with Re-hospitalization}

The Lasso model selected the following variables as important for rehospitalization: age, diabetes, need for dialysis, dyspnea, and bilateral infiltrates on presenting chest radiograph. Based on a univariate Cox proportional hazard model, age, diabetes, and dialysis requirement were associated with re-hospitalization; and dyspnea and bilateral infiltrates were inversely associated with re-hospitalization (Supplemental Table 4).

In a multivariable Cox proportional hazard regression analysis, older age (HR 1.01 per year [95\% CI 1.00-1.02]), diabetes (HR 1.54 [1.06-2.23]), and the need for dialysis (HR 3.78 [2.23-6.43]) during index presentation were statistically significantly associated with rehospitalization within 30 days of hospital discharge; and dyspnea (0.63 [0.44-0.91]) and bilateral infiltrates on presenting chest radiograph $(0.57$ [0.37-0.88]) remained inversely associated with rehospitalization within 30 days of discharge. In a sensitivity analysis including a subset of 970 patients with available COVID-19 PCR cycle threshold, the multivariable Cox proportional hazard regression analysis revealed similar results (Supplemental Table 5).

\section{Factors Associated with Mortality}

Based on a univariate Cox proportional hazard model, older age (1.09 [1.06-1.12]), Asian race (2.68 [1.17-6.14]), 

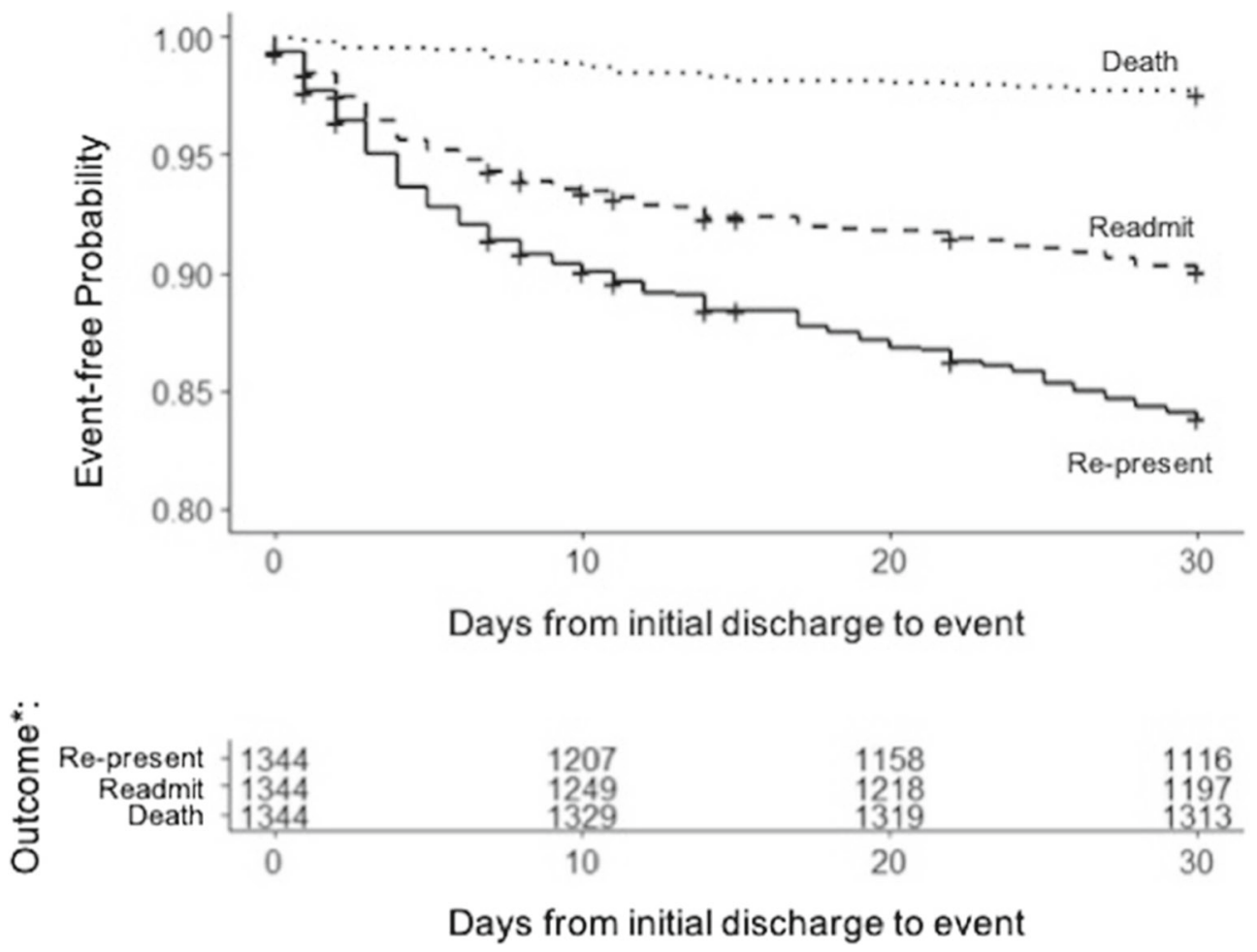

*Patients could experience more than one outcome.

Figure 2 Kaplan-Meier curve depicting 30-day post-discharge return the emergency room, re-hospitalization, and mortality of cohort patients.

hypertension (HR 3.46 [1.49-7.99]), diabetes (1.98 [0.993.99]), chronic kidney disease (2.93 [0.89-9.66]), and active malignancy (3.40 [1.31-8.82]) were significantly associated with death within 30 days of hospital discharge (Supplemental Table 6). Conversely, bilateral infiltrates on chest radiograph (0.25 [0.11-0.57]), higher body mass index (0.92 [0.86$0.98])$, presenting symptoms of fever $(0.51$ [0.25-1.03]), nausea $(0.26[0.06-1.08])$, myalgia $(0.33[0.10-1.10)$, and dyspnea $(0.33[0.16-0.68])$ were inversely associated with mortality.

In a multivariable Cox proportional hazard regression analysis, older age (HR 1.08 per year [95\% CI 1.05-1.11]) and Asian race (HR 2.89 [95\% CI 1.27-6.61]) were statistically significantly associated with mortality within 30 days of hospital discharge.

\section{DISCUSSION}

There were several important findings related to posthospitalization outcomes in this study of adults hospitalized with COVID-19 during the initial wave of the pandemic in NYC. We report that post-discharge return to an emergency department and re-hospitalization were common and identify determinants of adverse post-hospitalization outcomes, which may inform ongoing care of COVID-19 survivors.
Within 30 days of hospital discharge, nearly one in seven (16.1\%) patients returned to the ED, one in ten $(9.8 \%)$ were rehospitalized, and $2.4 \%$ had died. To our knowledge, this is the most complete study to date of 30-day post-hospitalization outcomes among COVID-19 infected patients, given our 5\% lost to follow-up rate and inclusion of post-hospitalization outcomes occurring both within and beyond our own health system. These data add to observations that symptoms of COVID-19 can persist for several weeks, ${ }^{12}$ and support the notion that the consequences of COVID-19 continue beyond hospital discharge. As was shown recently in a study from the Veterans Affairs healthcare system, the risk for adverse events is highest in the first 7-10 days following discharge. ${ }^{12}$ Our observations further demonstrate that risk persists beyond that early period and support the need for close follow-up after discharge. Moreover, our data show that risk is substantial whether the index event was an ED visit or a hospitalization. This supports the urgent need for protocols to assess patients after discharge (from either the ED or following hospitalization) for post-sequelae complications of COVID-19 and also provide necessary resources to optimize their care.

Importantly, our data also indicate that approximately onehalf of patients who either returned to the ED or were rehospitalized did so at a different hospital from the index presentation. Additionally, among patients who died, less than one-half $(46.9 \%)$ had been re-hospitalized and $6.2 \%$ had 
Table 2 Multivariable Cox Proportional Hazard Regression Analysis for Return to an Emergency Room, Re-hospitalization, and Mortality Within 30 Days of Index Hospital Discharge

\begin{tabular}{|c|c|c|}
\hline Variable & HR (CI) & $p$-value \\
\hline \multicolumn{3}{|l|}{ Return to an emergency room } \\
\hline Age (years) & $1.01(1.00-1.02)$ & 0.070 \\
\hline \multicolumn{3}{|l|}{ Sex } \\
\hline Male & Ref & - \\
\hline Female & $1.28(0.97-1.68)$ & 0.086 \\
\hline \multicolumn{3}{|l|}{ Race } \\
\hline White & Ref & - \\
\hline Asian & $1.15(0.77-1.72)$ & 0.494 \\
\hline Black & $1.01(0.68-1.50)$ & 0.954 \\
\hline Other & $0.95(0.65-1.38)$ & 0.777 \\
\hline Undomiciled & $5.13(2.83-9.3)$ & $<0.0001$ \\
\hline Dialysis required & $3.59(2.29-5.65)$ & $<0.0001$ \\
\hline Hospitalized vs ED discharge & $0.73(0.51-1.05)$ & 0.091 \\
\hline \multicolumn{3}{|l|}{ Infiltrates on initial chest radiograph } \\
\hline None & Ref & _ \\
\hline Unilateral & $0.75(0.49-1.13)$ & 0.162 \\
\hline Bilateral & $0.46(0.33-0.63)$ & $<0.0001$ \\
\hline \multicolumn{3}{|l|}{ Re-hospitalization } \\
\hline Age (years) & $1.01(1.00-1.02)$ & 0.021 \\
\hline \multicolumn{3}{|l|}{ Sex } \\
\hline Male & Ref & - \\
\hline Female & $1.11(0.78-1.58)$ & 0.563 \\
\hline \multicolumn{3}{|l|}{ Race } \\
\hline White & Ref & - \\
\hline Asian & $1.12(0.68-1.84)$ & 0.645 \\
\hline Black & $1.02(0.61-1.70)$ & 0.934 \\
\hline Other & $0.84(0.51-1.36)$ & 0.471 \\
\hline History of diabetes & $1.54(1.06-2.23)$ & 0.023 \\
\hline Dyspnea on arrival & $0.63(0.44-0.91)$ & 0.013 \\
\hline Dialysis required & $3.78(2.23-6.43)$ & $<0.0001$ \\
\hline Hospitalized vs ED discharge & $0.69(0.44-0.91)$ & 0.013 \\
\hline \multicolumn{3}{|l|}{ Infiltrates on initial chest radiograph } \\
\hline None & Ref & - \\
\hline Unilateral & $1.00(0.59-1.68)$ & 0.991 \\
\hline Bilateral & $0.57(0.37-0.88)$ & 0.012 \\
\hline \multicolumn{3}{|l|}{ Mortality } \\
\hline Age (years) & $1.08(1.05-1.11)$ & $<0.0001$ \\
\hline \multicolumn{3}{|l|}{ Sex } \\
\hline Male & Ref & _ \\
\hline Female & $1.70(0.81-3.59)$ & 0.200 \\
\hline \multicolumn{3}{|l|}{ Race } \\
\hline White & Ref & _ \\
\hline Asian & $2.89(1.27-6.61)$ & 0.012 \\
\hline Black & $0.31(0.04-2.45)$ & 0.300 \\
\hline Other & $0.99(0.34-2.89)$ & 0.999 \\
\hline
\end{tabular}

$* p<0.05$ in bold

returned to an ED but were not re-hospitalized. This likely explains the discrepancy observed between these data and a prior study reporting a 2-month readmission rate of just 9\%, which was based on electronic medical record review and thus may have led to an underestimation of re-hospitalization. ${ }^{14}$ Although the consequences of patients with COVID-19 returning to a different hospital are not clear, these findings further illustrate ongoing challenges in the USA with regard to fragmented care which has previously been shown to negatively impact health outcomes. ${ }^{27,}{ }^{28}$ Coupled with our finding that a substantial number of deaths occurred without return to the ED or a hospitalization, these data should additionally inform study design for future studies examining healthcare utilization and long-term COVID-19 sequelae.

Age and incident renal failure requiring dialysis were significantly associated with return to the ED and/or rehospitalization in the multivariable models. This was not surprising as age is one of the most important risk factors for adverse post-hospitalization outcomes in myriad other conditions (i.e., heart failure, stroke, and diabetes) ${ }^{29-32}$; and renal failure requiring dialysis may be suggestive of a complicated hospital course. Additionally, patients with diabetes were $\sim 50 \%$ more likely to be re-hospitalized within 30 days of discharge. Patients with these high-risk features likely warrant greater attention during the post-discharge period. While prediction tools assessing inpatient outcomes have been developed, ${ }^{33,34}$ our work suggests the possible utility of developing prediction tools that focus on post-hospitalization outcomes. Future study is also warranted to determine whether prioritizing such patients for follow-up in multidisciplinary COVID19 post-discharge clinics may be a fruitful strategy. ${ }^{35,36}$

Our observation that dyspnea and bilateral infiltrates on chest radiograph were inversely associated with returning to the ED and re-hospitalization was unexpected. We speculate that one possible explanation for this finding is that a classic, easily identifiable presentation in the setting of a novel viral syndrome, which can present in multiple varied ways, ${ }^{37-40}$ may have facilitated a more expedient diagnosis and perhaps more rapid treatment compared to those who presented in less typical ways. It is also possible that patients with more severe presentation (such as dyspnea and/or bilateral infiltrates on chest radiograph at presentation) received more aggressive treatment compared to those who did not have these features at presentation, which could have improved the outcomes among individuals who survived to discharge. Alternatively, it is possible that those presenting with classic symptoms have differing underlying pathophysiologic and/or immunologic mechanisms, therefore different outcomes. Future studies investigating these possibilities are needed.

There are important strengths and limitations to this study. To our knowledge, this is the first study of 30-day posthospitalization outcomes of COVID-19 survivors based on patient contact - this minimized loss-to-follow-up and ensured accurate data including utilization of healthcare services outside of our healthcare system network. Another important strength is that these data were derived from a comprehensive COVID-19 registry which included chart-level data on all consecutive symptomatic patients who presented to one of two hospitals in NYC. ${ }^{21}$ There are also several limitations. First, these data were collected from a specific region-future studies are needed to better understand patterns of posthospitalization outcomes in different areas of the country as clinical management may differ by region. Second, we did not have data on post-hospitalization processes such as the use of home health aides or whether patients had a follow-up ambulatory appointment - these aspects of care could have impacted outcomes. Finally, determinants of mortality should be interpreted with caution due to the relatively low number of events-future studies leveraging national cohorts will be necessary to better understand factors contributing to posthospitalization mortality. 
In conclusion, we report 30-day outcomes following hospital discharge after COVID-19 infection at two NYC hospitals, demonstrating that risk for adverse events from COVID-19 persists beyond the index presentation. COVID-19 infected adults merit close follow-up after discharge to fully optimize outcomes regardless of whether they are discharged from the emergency room or the hospital.

Supplementary Information The online version contains supple mentary material available at https://doi.org/10.1007/s11606-02106924-0

Acknowledgements: The authors would like to formally acknowl edge Dr. Martin Wells for his support and mentorship and Jason Benedict for data analysis consultation. We would also like to acknowledge our data abstractors, which included a team of Weill Cornell Medicine medical students and New York-Presbyterian/Weill Cornell Medical Center house staff: Zara Adamou BA, Haneen Aljayyousi BA, Bryan K. Ang BA, Elena Beideck BS, Orrin S. Belden MD/MBA, Anthony F. Blackburn BS, Joshua W. Bliss PharmD, Kimberly A. Bogardus BA, Chelsea D. Boydstun BA, Clare A. Burchenal MPH, Eric T. Caliendo BS, John K. Chae BA, David L. Chang BS, Frank R. Chen BS, Kenny Chen BA, Andrew Cho PhD, Alice Chung BA, Susana Martinez Diaz BA, Alisha N. Dua MRes, Andrew Eidelberg BS, Rahmi S. Elahiji BA, Mahmoud Eljalby MMSc, Emily R. Eruysal BS, Kimberly N. Forlenza MSc, Rana Khan Fowlkes BA, Rachel L. Friedlander BA, Gary George BS, Shannon Glynn BS, Leora Haber BA, Janice Havasy BS, Evan Honig BA, Alex Huang BA, Hao Huang BS, Jennifer H. Huang BS, Sonia Iosim BS, Julie Kim BS, Mitali Kini BS, Rohini V. Kopparam BS, Hannah Krinsky MS, Jerry Y. Lee BA, Justin Lee BS, Mark Lee BS BA, Aretina K. Leung BA, Bethina Liu AB, Charalambia Louka BS, Jessica Lu BA, Danny Luan MPH, Brienne Lubor BS, Dianne Lumaquin BS, Matthew L. Magruder BA, Ruth Moges MSc, Prithvi M. Mohan BS, Fatima Morales BA, Max F. Morin BS, Sophie Mou BA, J. J. Nario BS, Yuna Oh BS, Rachel Rosengard MSc, Noah Rossen BA, Emma M. Schatoff PhD, Pooja D. Shah BA, Sachin P. Shah BA, Daniel Skaf BS, Carolyn Stewart BA, Shoran Tamura BS, Ahmed Toure BA, Nahomy Ledesma Vicioso BS, Camila M. Villasante BA, Gal Wald BA, Samuel Williams BA, Ashley Wu BS, Andrew L. Yin BA, Lisa Zhang BA.

Corresponding Author: Justin R. Kingery, $M D / P h D$; Division of General Internal Medicine, Department of Medicine, Weill Cornell Medicine, New York, NY 10065, USA (e-mail: jrk9006@med.cornell. edu).

Funding This study received support from the Weill Cornell Clinical and Translational Science Center (CTSC) (UL1 TR000457). Dr. Kingery is supported by the National Institutes of Health/National Heart Lung Blood Institute grant K23-HL-152926. Dr. Choi is supported by the National Institutes of Health/National Center for Advancing Translational Sciences grant KL2-TR-002385.

\section{Declarations:}

Conflict of Interest: Dr. Safford has received research support from Amgen. Dr. Choi has received research support and consulting fees from Roche Diagnostics and Allergan unrelated to the work in this manuscript. Dr. Goyal has received personal fees for medicolegal consulting on heart failure.

The remaining authors have nothing to disclose.

\section{REFERENCES}

1. Dong E, Du H, Gardner L. An interactive web-based dashboard to track COVID-19 in real time. Lancet Infect Dis. Published online 2020 doi:https://doi.org/10.1016/S1473-3099(20)30120-1
2. Cutler DM, Summers LH. The COVID-19 Pandemic and the $\$ 16$ Trillion Virus. JAMA - J Am Med Assoc. Published online 2020. doi:https://doi. org/10.1001/jama.2020.19759

3. Wiersinga WJ, Rhodes A, Cheng AC, Peacock SJ, Prescott HC. Pathophysiology, Transmission, Diagnosis, and Treatment of Coronavirus Disease 2019 (COVID-19): A Review. JAMA - J Am Med Assoc. Published online 2020. doi:https://doi.org/10.1001/jama.2020.12839

4. Price-Haywood EG, Burton J, Fort D, Seoane L. Hospitalization and Mortality among Black Patients and White Patients with Covid-19. N Engl $J$ Med. Published online 2020. doi:https://doi.org/10.1056/ nejmsa2011686

5. Petrilli CM, Jones SA, Yang J, et al. Factors associated with hospital admission and critical illness among 5279 people with coronavirus disease 2019 in New York City: Prospective cohort study. BMJ. Published online 2020. doi:https://doi.org/10.1136/bmj.m1966

6. Cummings MJ, Baldwin MR, Abrams D, et al. Epidemiology, clinical course, and outcomes of critically ill adults with COVID-19 in New York City: a prospective cohort study. Lancet. Published online 2020. doi:https://doi.org/10.1016/S0140-6736(20)31189-2

7. Ackermann M, Verleden SE, Kuehnel M, et al. Pulmonary Vascular Endothelialitis, Thrombosis, and Angiogenesis in Covid-19. N Engl J Med. Published online 2020. doi:https://doi.org/10.1056/nejmoa2015432

8. Pan F, Ye T, Sun P, et al. Time Course of Lung Changes On Chest CT During Recovery From 2019 Novel Coronavirus (COVID-19) Pneumonia. Radiology. Published online 2020. doi:https://doi.org/10.1148/radiol. 2020200370

9. Hansen LO, Young RS, Hinami K, Leung A, Williams M V. Interventions to reduce 30-day rehospitalization: A systematic review. Ann Intern Med. Published online 2011. doi:https://doi.org/10.7326/0003-4819-155-8201110180-00008

10. Leppin AL, Gionfriddo MR, Kessler M, et al. Preventing 30-day hospital readmissions: A systematic review and meta-analysis of randomized trials. JAMA Intern Med. Published online 2014. doi:https://doi.org/10. 1001/jamainternmed.2014.1608

11. Dharmarajan $\mathbf{K}$, Wang $\mathbf{Y}$, Lin $\mathbf{Z}$, et al. Association of changing hospital readmission rates with mortality rates after hospital discharge. JAMA - J Am Med Assoc. Published online 2017. doi:https://doi.org/10.1001/ jama.2017.8444

12. Donnelly JPWXQITJPHC. Readmission and Death After Initial Hospital Discharge Among Patients With COVID-19 in a Large Multihospital System. JAMA. Published online 2020. doi:https://doi.org/10.1001/ jama.2020.21465

13. Chopra, Vineet; Flanders, Scott A; O'Malley, Megan; Malani, Anurag N; Prescott HC. Sixty-Day Outcomes Among Patients Hospitalized With COVID-19. Ann Intern Med. Published online 2020. https://doi.org/10. 7326/M20-5661

14. Lavery AM, Preston LE, Ko JY, et al. Characteristics of Hospitalized COVID-19 Patients Discharged and Experiencing Same-Hospital Readmission - United States, March-August 2020. MMWR Morb Mortal Wkly Rep. Published online 2020. doi:10.15585/mmwr.mm6945e2

15. Bowles KH, McDonald M, Barrón Y, Kennedy E, O'Connor M, Mikkelsen M. Surviving COVID-19 After Hospital Discharge: Symptom, Functional, and Adverse Outcomes of Home Health Recipients. Ann Intern Med. Published online 2020. doi:https://doi.org/10.7326/m205206

16. Parra LM, Cantero M, Morrás I, et al. Hospital readmissions of discharged patients with covid-19. Int $J$ Gen Med. Published online 2020. doi:https://doi.org/10.2147/IJGM.S275775

17. Jeon WH, Seon JY, Park SY, Oh IH. Analysis of risk factors on readmission cases of COVID-19 in the Republic of Korea: Using nationwide health claims data. Int J Environ Res Public Health. Published online 2020. doi:https://doi.org/10.3390/ijerph17165844

18. Atalla E, Kalligeros M, Giampaolo G, Mylona EK, Shehadeh F, Mylonakis E. Readmissions among patients with COVID-19. Int $J$ Clin Pract. Published online 2021. doi:https://doi.org/10.1111/ijcp. 13700

19. Somani SS, Richter F, Fuster V, et al. Characterization of Patients Who Return to Hospital Following Discharge from Hospitalization for COVID19. J Gen Intern Med. Published online 2020. doi:https://doi.org/10. 1007/s11606-020-06120-6

20. Ye S, Hiura G, Fleck E, et al. Hospital Readmissions After Implementation of a Discharge Care Program for Patients with COVID-19 Illness. $J$ Gen Intern Med. Published online 2021. doi:https://doi.org/10.1007/ s11606-020-06340-w

21. Goyal P, Choi JJ, Pinheiro LC, et al. Clinical Characteristics of Covid-19 in New York City. $N$ Engl $J$ Med. Published online 2020. doi:https://doi. org/10.1056/nejmc2010419 
22. Harris PA, Taylor R, Thielke R, Payne J, Gonzalez N, Conde JG. A metadata-driven methodology and workflow process for providing translational research informatics support, J Biomed Inform. J Biomed Inf Published online 2009.

23. Tibshirani R. The lasso method for variable selection in the cox model Stat Med. Published online 1997. doi:https://doi.org/10.1002/(SICI) 1097-0258(19970228)16:4<385::AID-SIM380>3.0.CO;2-3

24. Magleby R, Westblade LF, Trzebucki A, et al. Impact of Severe Acute Respiratory Syndrome Coronavirus 2 Viral Load on Risk of Intubation and Mortality Among Hospitalized Patients With Coronavirus Disease 2019. Clin Infect Dis. Published online 2020. doi:https://doi.org/10 1093/cid/ciaa851

25. van Buuren S, Groothuis-Oudshoorn K. mice: Multivariate imputation by chained equations in R. J Stat Softw. Published online 2011. doi:https://doi.org/10.18637/jss.v045.i03

26. White IR, Royston P, Wood AM. Multiple imputation using chained equations: Issues and guidance for practice. Stat Med. Published online 2011. doi:https://doi.org/10.1002/sim.4067

27. Kern LM, Rajan M, Ringel JB, et al. Healthcare Fragmentation and Incident Acute Coronary Heart Disease Events: a Cohort Study. J Gen Intern Med. Published online 2020. doi:https://doi.org/10.1007/s11606020-06305-Z

28. Pinheiro LC, Reshetnyak E, Safford MM, Nanus D, Kern LM. Healthcare fragmentation and cardiovascular risk control among older cancer survivors in the Reasons for Geographic And Racial Differences in Stroke (REGARDS) study. J Cancer Surviv. Published online 2020. doi:https:// doi.org/10.1007/s11764-020-00933-4

29. Tromp J, Bamadhaj S, Cleland JGF, et al. Post-discharge prognosis of patients admitted to hospital for heart failure by world region, and national level of income and income disparity (REPORT-HF): a cohort study. Lancet Glob Heal. Published online 2020. doi:https://doi.org/10. 1016/S2214-109X(20)30004-8

30. Brown AW, Therneau TM, Schultz BA, Niewczyk PM, Granger C V. Measure of Functional Independence Dominates Discharge Outcome Prediction after Inpatient Rehabilitation for Stroke. Stroke. Published online 2015. doi:https://doi.org/10.1161/STROKEAHA.114.007392

31. Lichtman JH, Leifheit-Limson EC, Jones SB, Wang Y, Goldstein LB Preventable readmissions within 30 days of ischemic stroke among medicare beneficiaries. Stroke. Published online 2013. doi:https://doi. org/10.1161/STROKEAHA.113.003165

32. Mirkin KA, Enomoto LM, Caputo GM, Hollenbeak CS. Risk factors for 30-day readmission in patients with congestive heart failure. Hear Lung $J$ Acute Crit Care. Published online 2017. doi:https://doi.org/10.1016/j. hrtlng.2017.06.005

33. Wongvibulsin S, Garibaldi BT, Antar AAR, et al. Development of Severe COVID-19 Adaptive Risk Predictor (SCARP), a Calculator to Predict Severe Disease or Death in Hospitalized Patients With COVID-19. Ann Intern Med. Published online 2021. doi:https://doi.org/10.7326/m206754

34. Garibaldi BT, Fiksel J, Muschelli J, et al. Patient Trajectories Among Persons Hospitalized for COVID-19 : A Cohort Study. Ann Intern Med. Published online 2021. doi:https://doi.org/10.7326/M20-3905

35. Sheehy LM. Considerations for postacute rehabilitation for survivors of COVID-19. J Med Internet Res. Published online 2020. doi:https://doi. org/10.2196/19462

36. Salawu A, Green A, Crooks MG, Brixey N, Ross DH, Sivan M. A proposal for multidisciplinary tele-rehabilitation in the assessment and rehabilitation of COVID-19 survivors. Int $J$ Environ Res Public Health. Published online 2020. doi:https://doi.org/10.3390/ijerph17134890

37. Guan W, Ni Z, Hu Y, et al. Clinical Characteristics of Coronavirus Disease 2019 in China. N Engl J Med. Published online 2020. doi:10.1056/ nejmoa2002032

38. Wu P, Duan F, Luo C, et al. Characteristics of Ocular Findings of Patients with Coronavirus Disease 2019 (COVID-19) in Hubei Province, China. JAMA Ophthalmol. Published online 2020. doi:https://doi.org/10.1001/ jamaophthalmol.2020.1291

39. Pan L, Mu M, Yang P, et al. Clinical characteristics of COVID-19 patients with digestive symptoms in Hubei, China: A descriptive, cross-sectional, multicenter study. Am J Gastroenterol. Published online 2020. doi:https://doi.org/10.14309/ajg.0000000000000620

40. Giacomelli A, Pezzati L, Conti F, et al. Self-reported olfactory and taste disorders in SARS-CoV-2 patients: a cross-sectional study. Clin Infect Dis. Published online 2020. doi:https://doi.org/10.1093/cid/ciaa330 